\title{
Evaluation of Linear Polymerization Shrinkage, Flexural Strength and Modulus of Elasticity of Dental Composites
}

\author{
Gabriela Queiroz de Melo Monteiro ${ }^{\natural *}$, Marcos Antonio Japiassú Resende Montes ${ }^{\mathrm{b}}$ \\ ${ }^{a}$ Curso de Odontologia do Centro Universitário de João Pessoa - UNIPÊ, \\ João Pessoa - PB, Brazil \\ ${ }^{\mathrm{b}}$ Departamento de Odontologia Restauradora, \\ Faculdade de Odontologia da Universidade de Pernambuco - FOP/UPE, \\ Camaragibe - PE, Brazil
}

Received June 25, 2009; Revised February 7, 2010

\begin{abstract}
Linear polymerization shrinkage (LPS), flexural strength (FS) and modulus of elasticity (ME) of 7 dental composites (Filtek Z350" ${ }^{\mathrm{TM}}$, Filtek Z250M/3M ESPE; Grandio ${ }^{\mathrm{TM}}$, Polofil Supra ${ }^{\mathrm{TM}} /$ VOCO; TPH Spectrum ${ }^{\mathrm{TM}}$, TPH3 ${ }^{\mathrm{TM}}$, Esthet-X $\mathrm{X}^{\mathrm{TM}} /$ Denstply) were measured. For the measurement of LPS, composites were applied to a cylindrical metallic mold and polymerized $(n=8)$. The gap formed at the resin/mold interface was observed using scanning electron microscopy $(1500 \times)$. For FS and ME, specimens were prepared according to the ISO 4049 specifications $(n=10)$. Statistical analysis of the data was performed with one-way ANOVA and the Tukey test. TPH Spectrum presented significantly higher LPS values $(29.45 \mu \mathrm{m})$. Grandio had significantly higher mean values for FS (141.07 MPa) and ME (13.91 GPa). The relationship between modulus of elasticity and polymerization shrinkage is the main challenge for maintenance of the adhesive interface, thus composites presenting high shrinkage values, associated with a high modulus of elasticity tend to disrupt the adhesive interface under polymerization.
\end{abstract}

Keywords: composite resin, modulus of elasticity, polymerization shrinkage, flexural strength

\section{Introduction}

Despite advances in adhesive dentistry, polymerization shrinkage of resin composites remains a challenge. Polymerization shrinkage is the consequence of molecular re-arrangement in a space smaller than the one needed during the liquid phase of the material; intermolecular distances may change from $0.3 \AA$ (van der Waals distance) to $0.1 \AA$ (covalent bonds). Resin composites polymerize as the consequence of a chemical reaction that involves the breakage of carbon double bonds and subsequent formation of simple carbon-carbon bonds, producing polymer chains. The consequence of this "molecular contraction" in the material is a volumetric loss of approximately $1.5 \% .^{1}$

Shrinkage promotes a deformation in the composite, which is clinically masked by the constraint of the bonded material, resulting in stress generation. ${ }^{2}$ As the reaction proceeds, the stress increases and eventually becomes higher than the adhesives bond strength, causing gap formation. ${ }^{3,4}$ In addition to marginal gap formation, stress at the tooth-restoration margins can lead to marginal discoloration, postoperative sensitivity and recurrent caries. These clinical consequences are the main reason for replacement of resin composites restorations and explain why polymerization shrinkage is the great limitation of these materials. ${ }^{3,5}$

The factors that influence the generation of stress are volumetric polymerization shrinkage, modulus of elasticity, visco-elastic behavior, adherence of the resin composite to the cavity walls and the configuration factor of the restoration (C-factor). ${ }^{3}$ The modulus of elasticity is a mechanical property that describes the relative stiffness or rigidity of a material and provides a direct relationship with the inorganic filler volume fraction. Modulus of elasticity is measured by the slope of the elastic region of the stress $\times$ strain diagram and has an important influence on the stress generated during shrinkage that accompanies the polymerization of resin composites. According to Hooke's law the strain of these materials produces stress. This law describes the linear relationship between stress and strain in an elastic solid. Since stress is a product of the modulus of elasticity by strain, materials with a combination of high volumetric shrinkage and high modulus of elasticity tend to generate high stresses during the polymerization reaction. ${ }^{6}$ In vitro studies suggest that polymerization stresses increase with increase in the stiffness of the resin composite. ${ }^{5}$

However, during the polymerization reaction, not all the shrinkage is converted into contraction stress, because the polymer is able to rearrange and relieve the generated stress. This is a result of the visco-elastic behavior, characterized by the flow capacity of composites in the early stages of the reaction and by the modulus of elasticity achieved during polymerization.

The mechanical properties of composites depend on many factors related to the composition of the materials, such as the type and quantity of the monomers used, their degree of conversion, type and size of the inorganic fillers, type of silanization and the amount of initiators present. The more inorganic filler added to the material, the smaller the total shrinkage and the higher the elastic modulus; however, this does not mean that a low shrinkage composite necessarily generates low shrinkage stress, because the increase in modulus of elasticity makes the material less capable of undergoing plastic deformation during shrinkage, diminishing the possibility of stress relief. ${ }^{7}$

In order to enhance the physical, chemical and mechanical properties of resin composites, some changes have been proposed: the incorporation of new monomers, new initiation systems and new technologies, such as nanotechnology, for inorganic filler production. Nanotechnology consists of the production of materials and functional structures in an interval between 0.1 and $100 \mathrm{~nm}$, the nanoscale, using various physical and chemical methods. ${ }^{8-10}$

Within a certain limit, the smaller the size of the inorganic filler the more fillers can be incorporated into the resin composite. An increase in inorganic filler concentrations, although favoring decreases 
in polymerization shrinkage, promotes increases in the modulus of elasticity. The relationship between modulus of elasticity and polymerization shrinkage values is a way to predict stress generation at the adhesive interface. Nevertheless, whether the incorporation of nanometric inorganic fillers promotes an increase in the physical properties of resin composites, such as flexural strength and modulus of elasticity, and whether it promotes a reduction of the composites polymerization shrinkage, remains uncertain.

\section{Materials and Methods}

The materials, manufacturers, compositions and batch numbers for this study are listed in Table 1 .

\subsection{Linear polymerization shrinkage}

The restorative composites were placed in a circular metallic mold (inner diameter $7 \mathrm{~mm}$, height $2 \mathrm{~mm}$ ). The composites were then covered with a mylar strip and pressed with a microscope glass slab. Eight specimens were prepared for each material. Photo-activation was performed with the curing tip positioned close to the metallic mold/restorative composite for 40 seconds, with light intensity of $600 \mathrm{~mW} . \mathrm{cm}^{-2}$ using an Optilight Plus ${ }^{\mathrm{TM}}$ curing unit (Gnatus, Ribeirão Preto, São Paulo, Brazil). The light intensity was measured with a radiometer (Gnatus, Ribeirão Preto, São Paulo, Brazil). After 15 minutes, the top and bottom surfaces of the specimens were polished with sandpaper of decreasing grit (nos. 320, 600, 1200) and then placed in an ultrasound cube for 2 minutes. After 24 hours at $37^{\circ} \mathrm{C}$, the specimens were mounted on stubs, gold sputtered (sputter coater SCD 050 Bal-Tec, Schaan, Liechtenstein) and, using scanning electron microscopy (JEOL JSM-6360 SEM, Japan; 1500×), the gap formed between the metallic mold and the resin composite was observed at 4 points located in positions corresponding to 3,6, 9 and 12 hours of a clock face. ${ }^{11}$ Images were taken and the gaps were measured (in micrometers) using the ImageJ program (Image Processing and Analysis in Java). ${ }^{12}$ The arithmetic means were calculated for each specimen.

\subsection{Flexural strength and modulus of elasticity}

Flexural strength was determined according to the International Organization for Standardization (ISO) Standard 4049. ${ }^{13}$ Resin com- posites were inserted in a split stainless steel mold $(25 \times 2 \times 2 \mathrm{~mm})$, covered with a mylar strip and a glass microscope slab, then lightcured using the Optilight Plus curing unit described above. The light curing procedure was performed with 3 overlapping sections of 40 seconds each. This procedure was repeated on the opposite side. Ten specimens were prepared for each group. Fifteen minutes after light curing, the specimens were then freed from the mold, gently wet-ground (sandpaper paper no. 320) to remove any flash, and transferred to a distilled water bath at $37^{\circ} \mathrm{C}$. After 24 hours, the dimensions of the specimens were measured to an accuracy of $0.01 \mathrm{~mm}$ using a digital micrometer. The 3-point bend fixture consisted of 2 rods (diameter $2 \mathrm{~mm}$ ) mounted parallel with $20 \mathrm{~mm}$ between their centers (support span $20 \mathrm{~mm}$ ). Each specimen was loaded at its center with a $2 \mathrm{~mm}$ diameter striker at a cross-head speed of $0.5 \mathrm{~mm} / \mathrm{min}$ until failure (Universal Testing Machine, Kratos Dynamometers, São Paulo, Brazil). Flexural strength $(\sigma)$ and modulus of elasticity $(E)$ were calculated using the following formulas:

$$
\begin{aligned}
& \sigma=3 P L / 2 b h^{2}(\text { measured in } \mathrm{MPa}) \\
& E=F L^{3} / 4 b h^{3} d \times 10^{-3}(\text { measured in } \mathrm{GPa})
\end{aligned}
$$

where $L$ is the distance between the supports (fixed at $20 \mathrm{~mm}$ ), $b$ is the specimen width ( $\mathrm{mm}), h$ is the specimen height $(\mathrm{mm}), F$ is the load $(\mathrm{N})$ at a convenient point on the straight line portion of the curve, $d$ the deflection ( $\mathrm{mm}$ ) at load $F$, and $P$ is the maximum load $(\mathrm{N})$ resulting in failure.

Statistical evaluation of the data was performed by one-way analysis of variance (ANOVA) and the Tukey test; a 5\% significance level was used $(p=0.05)$.

\section{Results}

The results for the linear polymerization shrinkage are shown in Figure 1. The highest value for the coefficient of variation was $31.82 \%$ (Esthet-X). ANOVA rejected the null hypothesis, showing that at least one of the groups differed from the rest $(p=0.00001)$. The Tukey paired comparisons test, at $5 \%(p<0.05)$, demonstrated statistically significant differences between TPH Spectrum and all the other groups, as shown by the different letters in parentheses.

\begin{tabular}{|c|c|c|c|}
\hline Composite & Matrix $^{a}$ & $\begin{array}{l}\text { Filler content; filler size; } \\
\quad \% \text { in mass/volume }\end{array}$ & $\begin{array}{l}\text { Manufacturer; } \\
\text { batch no. }\end{array}$ \\
\hline Filtek Z350 & $\begin{array}{l}\text { BisGMA, UDMA, TEGDMA } \\
\text { and BisEMA }\end{array}$ & Zirconia/silica and silica; nanoparticle; 78.5/59.5 & 3M/ESPE; 6EB \\
\hline Filtek Z250 $0^{\mathrm{TM}}$ & BisGMA, UDMA and BisEMA & Zirconia/silica; microhybrid; $82 / 60^{11}$ & 3M/ESPE; $5 \mathrm{WK}$ \\
\hline Grandio $^{\mathrm{TM}}$ & BisGMA and TEDMA & -; nanohybrid; 87/71.4 & VOCO; 621307 \\
\hline Polofil Supra ${ }^{\mathrm{TM}}$ & BisGMA, UDMA and TEDMA & -; microhybrid; 76.5/60 & VOCO; 571239 \\
\hline Esthet-X $\mathrm{XM}^{\mathrm{TM}}$ & $\begin{array}{l}\text { U-BisGMA, BisEMA and } \\
\text { EGDMA }\end{array}$ & $\begin{array}{l}\text { Boro-silicate/aluminium/barium glass and silica; } \\
\text { nanohybrid; 77/_-11 }\end{array}$ & $\begin{array}{l}\text { Dentsply/Caulk; } \\
0510082\end{array}$ \\
\hline TPH $3^{\mathrm{TM}}$ & $\begin{array}{l}\text { BisGMA and BisEMA } \\
\text { dimethacrylate }\end{array}$ & $\begin{array}{l}\text { Boro-silicate/aluminium/barium glass and silica; } \\
\text { nanohybrid; 75.27/- }\end{array}$ & $\begin{array}{l}\text { Dentsply/Caulk; } \\
492008\end{array}$ \\
\hline TPH Spectrum ${ }^{\mathrm{TM}}$ & U-BisGMA & $\begin{array}{l}\text { Boro-silicate/aluminium, barium and silica; } \\
\text { microhybrid; } 77 / 57.1^{11}\end{array}$ & $\begin{array}{l}\text { Dentsply/Caulk; } \\
541552\end{array}$ \\
\hline
\end{tabular}

Table 1. Composition, Manufacturers and Batch numbers of the materials studied.

All composites were A3 color. -, information not available from the manufacturer.

aBisGMA, bisphenol-glycidyl methacrylate; UDMA, urethanethyl dimethacrylate; TEGDMA, triethylene glycol dimethacrylate; BisEMA, bisphenolpolyethylene glycol dimethacrylate; U-BisGMA, urethane modified bisphenol-glycidyl methacrylate.

'3M ESPE, St. Paul, MN, USA; Dentsply Caulk, Milford, DE, USA; VOCO, Cuxhaven, Germany. 


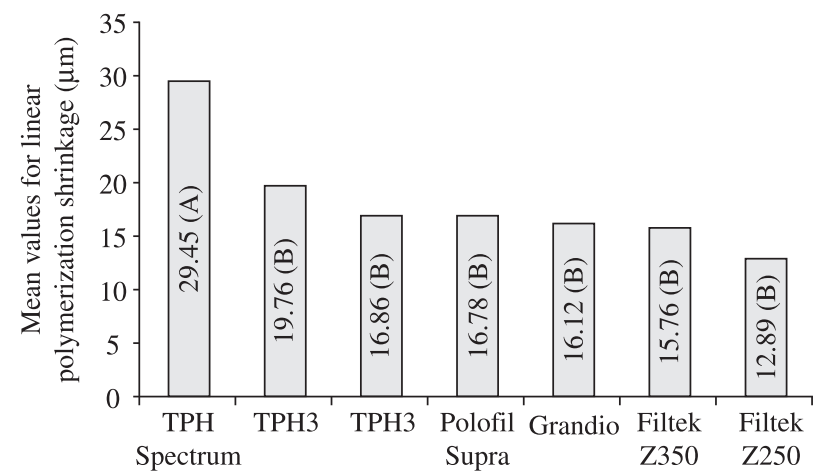

Figure 1. Results for linear polymerization shrinkage.

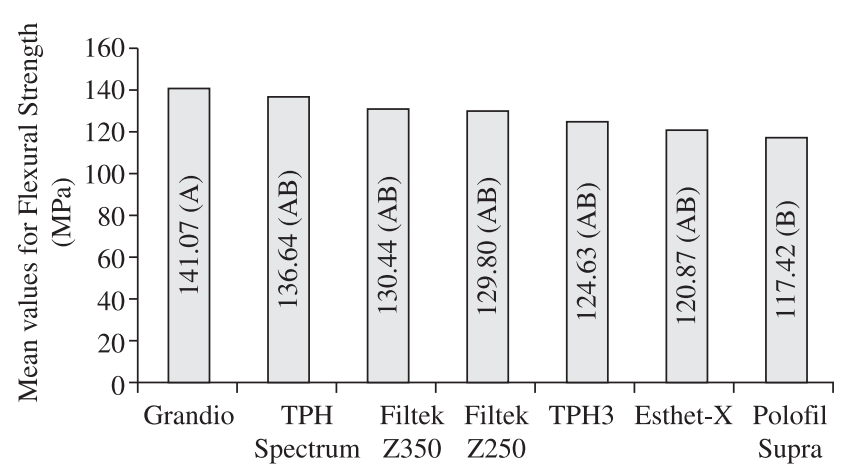

Figure 2. Results for flexural strength test.

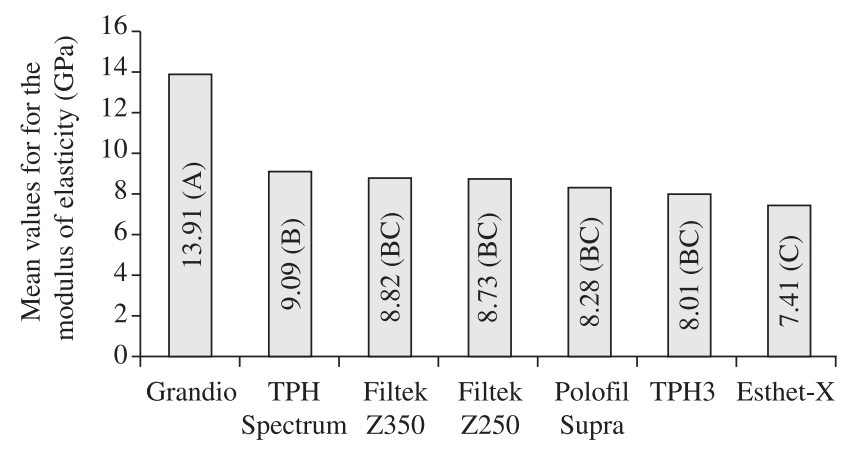

Figure 3. Results for modulus of elasticity.

Figure 2 shows that flexural strength varied from $117.42 \mathrm{MPa}$ (Polofil Supra) to $141.07 \mathrm{MPa}$ (Grandio), with significant differences existing between these groups $(p=0.0188)$. The highest coefficient of variation was $21.31 \%$ (Filtek Z250).

Results for the modulus of elasticity are shown in Figure 3. Values varied from 7.41 GPa (Esthet-X) to $13.91 \mathrm{GPa}$ (Grandio). The highest coefficient of variation was $15.52 \%$ (Esthet-X). Statistically significant differences were found between Grandio and all the other groups and between TPH Spectrum and Esthet-X ( $p=0.0001)$.

\section{Discussion}

Reducing polymerization shrinkage is a primary goal towards diminishing stress generation at the bonded interface. On the other hand, assessing the true shrinkage remains a challenge. Several methods can be used for such measurements, and for each one, a distinct aspect of polymerization shrinkage is measured. ${ }^{14}$
To measure volumetric shrinkage, multi-axial dimensional changes are accessed. However, considering the difficulty in accomplishing these measurements, linear changes are frequently measured since volumetric deformation can be mathematically derived from linear values. Shrinkage measurements can also record the total shrinkage (i.e. pre- and post-gel shrinkage) or only post-gel contraction. The latter requires a sensor that ignores viscous flow and is sensitive only to shrinkage of a solid with measurable stiffness. ${ }^{15}$

If the degree of polymerization is not taken into account, volumetric shrinkage is mainly dependant on the chemical composition of the resin matrix and the percentage of filler load, while the contraction stress and flow of the resin-based material is determined by Young's modulus of the composite resin. ${ }^{16}$

\subsection{Linear polymerization shrinkage}

The highest values in the linear polymerization shrinkage test were for TPH Spectrum $(29.45 \mu \mathrm{m})$ and this result was statistically significant in comparison to the other groups $(p=0.0001)$. There was a tendency towards a smaller shrinkage for the Filtek Z250 composite $(12.09 \mu \mathrm{m})$. The groups with the lowest shrinkage (Filtek $\mathrm{Z} 250=12.09 \mu \mathrm{m}$, Filtek $\mathrm{Z} 350=15.76 \mu \mathrm{m}$ and Grandio $=16.12 \mu \mathrm{m})$ were also those with the highest percentage of inorganic fillers in weight/volume (82/60\%, 78.5/59.5\% and 87/71.4\%, respectively).

The high polymerization shrinkage observed in for TPH Spectrum could be attributed to its composition. TPH Spectrum contains $77.5 \%$ of inorganic filler by weight, and $57.1 \%$ by volume, ${ }^{17}$ which is the smallest percentage of filler by volume within the composites studied for which compositional data were available (Dentsply does not supply this information).

In addition to the concentration, the shape of the inorganic fillers can also influence polymerization shrinkage. Shrinkage may be greater for composites with irregular shape due to the low interaction between the particles. ${ }^{18}$ Previous studies have reported that the TPH Spectrum resin composite has irregular-shaped fillers and that the zirconia/silica fillers present in composites manufactured by 3M/ESPE (Z100, Filtek Z250, Filtek P60 and Filtek flow) are round-shaped. ${ }^{19}$ Some advantages have been attributed to zirconia/ silica fillers. First, the microstructure of these fillers tends to absorb energy, making crack propagation difficult. Second, its chemical characteristics allow efficient coupling of the cover system (silane), improving the filler-matrix union, and the large and homogeneous distribution allows greater incorporation of filler. In contrast, materials with bimodal distribution, which combines barium glass and extremely thin amorphous silica, tend to be more sticky and hard to manipulate due to the greater voids between the particles. In addition, round-shaped fillers allow the formulation of materials with high filler concentration, without sacrificing manipulation properties..$^{20}$ These fillers also tend to increase resistance to wear and fracture, since there is no concentration of mechanical stress, due to the absence of angles and protuberances. ${ }^{10}$

However, evaluation of the concentrations and the shapes of the fillers used do not fully explain our results, leading us to analyze the influence of monomer composition on polymerization shrinkage. ${ }^{21}$ The composition of TPH Spectrum is based only on urethane modified BisGMA; this monomer corresponds to a BisGMA molecule with urethane bonds that tend to lower the viscosity of BisGMA, raising the mobility of its molecules during the polymerization of the organic matrix and, possibly, increasing the degree of conversion and leading to higher polymerization shrinkage. ${ }^{11}$ Specific tests (i.e. Raman spectroscopy) should be performed to prove this hypothesis. The monomeric composition of Filtek Z250 and Filtek Z350 is BisGMA, UDMA and BisEMA, all of which are high molecular weight monomers with high viscosity and lower polymerization 
shrinkage. BisEMA, for example, corresponds to a BisGMA molecule without the hydroxyls. In addition to the 3 base monomers, the Filtek Z350 composite contains the diluent monomer, TEGDMA, probably because this is a nanoparticule composite, requiring a certain dilution of the base monomers to incorporate filler particles without altering the composite's viscosity. However, it appears that the incorporation of this diluent monomer did not affect the increase in polymerization shrinkage.

\subsection{Flexural strength test}

The results from the 3-point bending test showed statistically significant differences ( $p=0.0188$ ) between Grandio (141.07 MPa) and Polofil Supra (117.42 MPa) composites. Even though these 2 resin composites are from the same manufacturer (VOCO), they have different chemical compositions, with regard to the filler size and the base monomers used. Grandio contains BisGMA and TEDMA, and Polofil Supra contains UDMA in addition to these 2 monomers.

Studies on the influence of monomer composition on the mechanical properties of resin composites have found that flexural strength increases when BisGMA or TEGDMA are substituted by UDMA. Furthermore, a reduction in flexural strength was observed when BisGMA was substituted by TEGDMA, showing that the degree of conversion does not necessarily have a positive correlation with all the mechanical properties. ${ }^{22}$ This is an isolated factor that could explain our results. Other factors such as filler size and composition, amount of initiators and the quality of silanization can also contribute to the development of physical and mechanical properties.

The high flexural strength values achieved with Grandio could be also be explained by the fact that, of the composites studied, Grandio has the highest filler concentration. This high concentration favors the development of mechanical properties such as compressive strength, hardness, flexural strength and modulus of elasticity, and also tends to reduce polymerization shrinkage, ${ }^{10,21}$ however, such a direct relationship between filler concentration and flexural strength has not been shown by other authors. ${ }^{17}$

The use of standardized protocols, such as ISO 4049, allows the results from different studies to be compared. Despite such standardization, these data demonstrate great variation, which is perhaps not surprising given the presence of some variables that can influence the results such as exposure time, light intensity and even the use of light boxes instead of conventional light units. Moreover, limitations still exist when trying to extrapolate these results to the clinical performance of materials, since the ISO 4049 standards for flexural strength testing recommend submerging the specimen in distilled water for only 24 hours prior to testing; furthermore, the specimen is submitted to only one mechanical cycle before submitting to fracture. These specifications do not reflect the material's long-term performance. Despite these considerations, the 3-point bending test used according to ISO 4049 is still considered a standard test. ${ }^{17}$

\subsection{Modulus of elasticity}

The results obtained for the modulus of elasticity demonstrate statistically significant differences between the groups studied. Grandio (13.91 GPa) was statistically different from all the other groups and TPH Spectrum (9.09 GPa) was statistically different from Esthet-X (7.41 GPa). These results are in accordance with other findings, where composites with high filler content had a high modulus of elasticity, and Grandio resin composite showed the highest modulus. ${ }^{10,23}$

In addition to the intimate relationship between filler content and the modulus of elasticity, as described above, the composition of the organic matrix also influences this property. In this study, this relationship can be observed from the differences found between the composites from the same manufacturer (Dentsply), Esthet-X and TPH Spectrum. These composites differ in filler size (Esthet-X 0.01-0.08 $\mu \mathrm{m}$; TPH Spectrum 0.04-5 $\mu \mathrm{m}$ ) and monomer composition. Despite the differences in size, the percentages and type of particles are very similar, leading to the conclusion that differences in monomer composition were responsible for the discrepant values in the modulus of elasticity. TPH Spectrum contains urethane modified BisGMA only, and Esthet-X contains BisEMA and TEGDMA. The presence of TEGDMA in Esthet-X could be the reason for the low modulus of elasticity values. This diluent monomer tends to decrease the stiffness of the polymer network and, consequently, the modulus of elasticity. A discrete increase in the modulus of elasticity was found when BisGMA was substituted by TEGDMA, followed by a significant decrease as the TEGDMA concentration increased. ${ }^{22}$

The low strength in composites with high TEGDMA concentrations can be caused by the tendency that this small and flexible monomer has for cyclization, instead of cross-linking. The pendent double bonds react to form primary cycles, which increase the degree of conversion, but do not contribute to the formation of the polymeric chain. Cross-linking is important for the formation of a good polymer chain and, consequently, good mechanical properties. ${ }^{24}$

The correlation between the data obtained by linear polymerization shrinkage and modulus of elasticity is extremely important to partially understand the behavior of resin composites during the polymerization reaction. Composites with low linear polymerization shrinkage, associated with a high modulus of elasticity (Grandio), although polymerizing to form small gaps, tend to create a rigid contraction; that is, they develop high stress at the bonding interface as a result of the contraction that occurs during polymerization. This contraction is attributed to the high stiffness of the material which makes stress relief through plastic deformation difficult. In addition, composites with high polymerization shrinkage and high modulus of elasticity (TPH Spectrum) will have even worse effects at the toothrestoration interface, overcoming the adhesive bond strength; this may lead to the development of fracture lines in the enamel, at the restoration margins and, more rarely, fracture of the cusps weakened by preparation.

The influence of the modulus of elasticity of composites on the stress generated during linear polymerization shrinkage has been studied previously by Pereira et al. ${ }^{1}$ In this investigation, the polymerization shrinkage of 4 commercial resin composites (Filtek Z250/3M ESPE, Filtek Supreme/3M ESPE, Admira/VOCO and Grandio/VOCO) were evaluated using 3 different methods: the magnitude of the stress generated during polymerization, linear polymerization shrinkage and wall-to-wall shrinkage in cylindrical cavities of bovine dentin; gaps in the second and third methods were examined by optical microscopy. The results showed that there were no significant differences in the magnitude of the stress generated; the Grandio composite had the highest values. With regard to the linear polymerization shrinkage, statistically significant differences were observed between the composites evaluated, with the Grandio composite presenting the smallest gap. In the wall-to-wall evaluation, there were also no statistically significant differences between the groups studied; these results suggest that materials with high filler content and high modulus of elasticity have smaller polymerization shrinkage when free to contract. However, when in constrained conditions, for example, when bonded to the cavity walls, making contraction difficult, these materials may generate considerably high stresses in relation to their own shrinkage.

Similar results were observed for all the tests performed in this study for the resin composites, Filtek Z350 and Filtek Z250, with only numerical differences. These results confirm that nanoparticle resin composites perform similarly to universal resin composites (hybrids). ${ }^{10}$ 
Recently siloranes (derived from the combination of siloxanes and oxiranes) have been proposed as low-shrinkage dental composites. ${ }^{16}$ The low polymerization shrinkage is due to the ring-opening oxirane monomer. ${ }^{25}$ As a result, the new silorane-based material has the ability to compensate for shrinkage by opening the oxirane ring during polymerization. ${ }^{26}$ This material seem to have comparable properties and slightly reduced shrinkage compared with Bis-GMAbased materials, thus probably representing a valid alternative to dimethacrylates. ${ }^{27}$

Depending on the intended use, composites with different mechanical properties may be required; in some situations the materials must be hard and strong, while in other situations the flexibility of the material is more important and strength is not an important factor; in contrast, stiffness or resilience may be the properties of interest. ${ }^{22}$ Hence, the physical and mechanical properties of resin composites depend on many variables of which clinicians should be aware.

The relationship between polymerization shrinkage and modulus of elasticity suggests that composites with low shrinkage, although associated with a high modulus of elasticity, will tend to generate high stresses at the bonded interface due to rigid contraction. Furthermore, composites with high shrinkage and high modulus will tend to produce even higher shrinkage stresses, risking the integrity of the bonded interface.

Future work measuring shrinkage stress and correlating the results with modulus of elasticity will help to confirm this hypothesis. Still, accessing polymerization shrinkage through different methods should also be performed since the results tend to vary especially between methods.

\section{Acknowledgements}

This research was supported by CAPES (Coordenação de Aperfeiçoamento de Pessoal de Nível Superior, Ministry of Education, Brazil).

\section{References}

1. Pereira RA, Araújo PA, Casteñeda-Espinosa JC and Mondelli RFL. Comparative analysis of the shrinkage stress of composite resins. Journal of Applied Oral Science. 2008; 16(1):30-34.

2. Gayosso CA, Santana FB, Ibarra JG, Espínola GS and Martinez MAC. Calculation of contraction rates due to shrinkage in light-cured composites. Dental Materials. 2004; 20(3):228-235.

3. Peutzfeldt A and Asmussen E. Determinants of in vitro gap formation of resin composites. Journal of Dentistry. 2004; 32(2):109-115.

4. Park JW and Ferracane JL. Measuring residual stress in dental composites using a ring slitting method. Dental Materials. 2005; 21(9):882-889.

5. Kleverlaan CJ and Feilzer AJ. Polymerization shrinkage and contraction stress of dental resin composites. Dental Materials. 2005; 21(12):1150-1157.

6. Helvatjoglu-Antoniades M, Papadogiannis Y, Lakes RS, Dionysopoulos $\mathrm{P}$ and Papadogiannis D. Dynamic and static elastic moduli of packable and flowable composite resins and their development after initial photo curing. Dental Materials. 2006; 22(5):450-459.

7. Ferracane JL. Developing a more complete understanding of stresses produced in dental composites during polymerization. Dental Materials. 2005; 21(1):36-42.
8. Mitra SB, Wu D and Holmes BN. An application of nanotechnology in advanced dental materials. Journal of the American Dental Association. 2003; 134(10):1382-1390.

9. Braga RR, Ballester RY and Ferracane JL. Factors involved in the development of polymerization shrinkage stress in resin-composites: a systematic review. Dental Materials. 2005; 21(10):962-970.

10. Beun S, Gloriex T, Devaux J, Vreven J and Leloup G. Characterization of nanofilled compared to universal and microfilled composites. Dental Materials. 2007; 23(1):51-59.

11. Obici AC, Sinhoreti MAC, Goes MF, Consani S and Sobrinho LC. Effect of photo-activation method upon polymerization shrinkage of restorative composites. Operative Dentistry. 2002; 27(1):192-198.

12. Rasband WS. ImageJ. [online]. Available from: http://rsb.info.nih.gov/ ij/,1997-2006. Access in: july 15, 2006.

13. ISO Standards. ISO 4049 Dentistry - polymer-based filling, restorative and luting materials. 3rd edition. Geneva: International Organization for Standardization; 2000. p. 1-22.

14. Sakaguchi RL, Wiltbank BD and Shah NC. Critical configuration analysis of four methods for measuring polymerization shrinkage strain of composites. Dental Materials. 2004; 20(4):388-396.

15. Watts DC and Satterthwaite JD. Axial shrinkage-stress depends upon both C-factor and composite mass. Dental Materials. 2008; 24(1):1-8.

16. Cadenaro M, Marchesi G, Scuor N, Breschi L, Davidson CL and Di Lenarda R. Assessment of polymerization contraction stress of three composite resins. Dental Materials. 2008; 24(5):649-654.

17. Walker MP, Haj-Ali R, Wang Y, Hunziker D and Williams KB. Influence of environmental conditions on dental composite flexural properties. Dental Materials. 2006; 22(11):1002-1007.

18. Adabo GL, Cruz CAS, Fonseca, RG and Vaz LG. The volumetric fraction of inorganic particles and the flexural strength of composites for posterior teeth. Journal of Dentistry. 2000; 31(5):353-359.

19. Sabbagh J, Ryelandt L, Bacherius L, Biebuyck JJ, Vreven J, Lambrechts $\mathrm{P}$ and Leloup G. Characterization of the inorganic fraction of resin composites. Journal of Oral Rehabilitation. 2004; 31(11):1090-1101.

20. Kaufman G. A new resin composite restorative incorporating synthetic filler technology and optimized particle size distribution. Esthetic Dentistry Update. 1994; 5:10-12.

21. Cadenaro M, Marchesi G, Antoniolli F, Davidson C, Dorigo EDS and Breschi L. Flowability of composites is no guarantee for contraction stress reduction. Dental Materials. 2009; 25(5):649-654.

22. Asmussen E and Peutzfeldt A. Influence of UEDMA, BisGMA and TEGDMA on selected mechanical properties of experimental resin composites. Dental Materials. 1998; 14(1):51-6.

23. Mesquita RV, Axmann D and Geis-Gerstorfer J. Dynamic visco-elastic properties of dental composite resins. Dental Materials. 2006; 22(3):258-267.

24. Floyd CJE and Dickens SH. Network structure of Bis-GMAand UDMA-based resin systems. Dental Materials. 2006; 22(12):1143-1149.

25. Weinmann W, Thalacker $\mathrm{C}$ and Guggenberger R. Siloranes in dental composites. Dental Materials. 2004; 21(1):68-74.

26. 3M ESPE. Filtek LS. Technical Profile. Available from: http:// solutions.3m.com/wps/portal/3M/en_US/3M-ESPE/dental-professionals/ products/category/direct-restorative/filtek-ls. Access in: 2009

27. Ilie $\mathrm{N}$ and Hickel R. Macro-, micro- and nano-mechanical investigations on silorane and methacrylate-based composites. Dental Materials. 2009; 25(6):810-819. 
